\title{
A Three Case Series of Neuroimaging Findings of Patients with Tuberous Sclerosis Complex
}

\author{
${ }^{1}$ Dr. Nihaal Reddy, ${ }^{2}$ Dr. Hemanth Kumar, ${ }^{3}$ Dr.Harpreet Singh,,${ }^{4}$ Dr.Ashwin Patil \\ ${ }^{1}$ (Department of Radiodiagnosis, KLE University, India) \\ ${ }^{2}$ (Department of Radiodiagnosis, KLE University, India) \\ 3 (Department of Radiodiagnosis, KLE University, India) \\ ${ }^{4}$ (Department of Radiodiagnosis, KLE University, India)
}

\begin{abstract}
:
Aim and Objective: To study different imaging findings in patients presenting with tuberous sclerosis

Materials \&Methods: We present neuroimaging findings in three children with tuberous sclerosis showing different brain findings on MRI and ct brain scans.

Result:We report the neuroimaging findings of 3 cases, two aged 12 years and one aged 2 years who clinically presented with delayed development and epilepsy with findings of subependymal giant cell astrocytoma, calcified tubers, transmantile cerebral white matter migration lines, subependymal nodules and cerebral atrophy.

Conclusion: Tuberous sclerosis also known as Bourneville pringle disease is an autosomal dominant genetic disease that is characterised by the presence of multiple hamartomas in various organs.

Two separate genes have been reported that have been mutated or deleted kin patients with TSC. TSC1 gene codes for the protein hamartin and TSC2 which codes for protein tuberin.

Neuroimaging plays an important role in making the diagnosis of TSC as around 95\% of affected patients show abnormalities in the brain.
\end{abstract}

Keywords: Tubers, astrocytoma, subependymal nodules, atrophy, transmantile lines

\section{Introduction:}

Tuberous sclerosis complex(TSC) is an autosomal dominant genetic disease that is characterised by presence of hamartomas in multiple organ systems.

Classically, TSC has been characterized by clinical triad of mental retardation, epilepsy, and skin lesions known as adenoma sebaceum. Though recent studies have shown that half of affected patients have normal intelligence, with $75 \%$ having epilepsy, with almost any organ of body being affected.

Neuroimaging plays an important role in making the diagnosis of TSC, as the characteristics abnormalities are present in about $95 \%$ of the cases.

The neuroimaging findings are usually present before the time of the birth, whereas other clinical findings like adenoma sebaceum develop later on in childhood.

The common neuroimaging abnormalities found in TSC are cortical tubers, subependymal nodules, subependymal giant cell astrocytoma, white matter migration lines, and cerebral cortical dysplasias.

Radiological confirmation has been proved to be sufficient for the confirmation of the lesions.

Case 1

\section{Results}

A 10 year old female child born to non-consanguineous parents

\section{Clinical Profile:}

Child cried immediately after birth with no history of intensive care stay.

Development was normal till age of 3 years, regressed since then with child presenting now with epilepsy and mental retardation.

Imaging: MRI showed multiple T2 hyperintense areas in the posterior bilateral peritrigonal regions and fronto-parietal subcortical white matter suggesting cortical tubers or hamartomas

CT scan revealed multiple subependymal and periventricular calcifications. 
Fig 1a, b,c,d) multiple hyperintense cerebral tubers ( arrows) seen on FLAIR and T2 weighted images
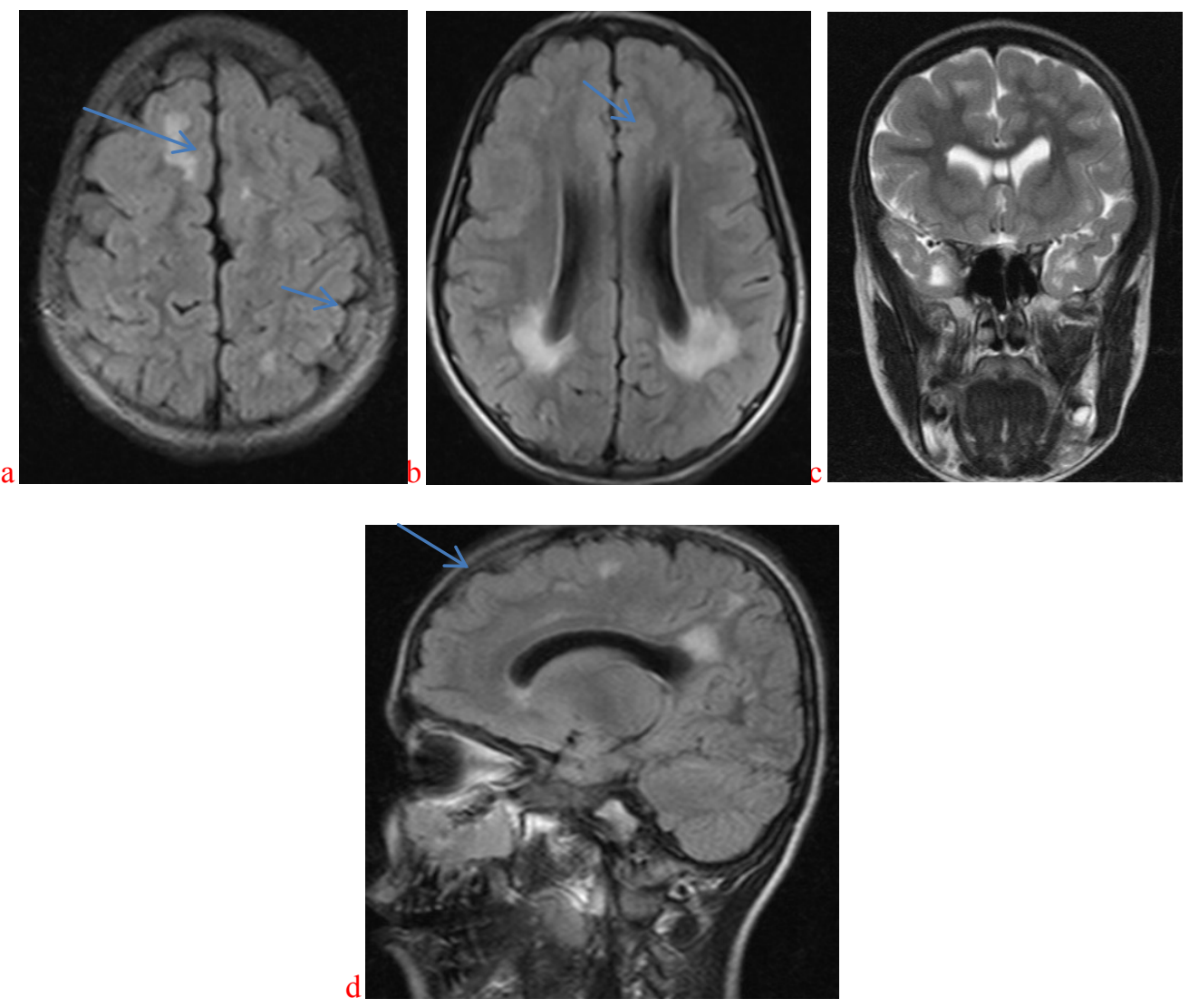

\section{Case 2}

A 2 year old female child born to consanguineous parents

ClinicalProfile:

Child had an uneventful birth history, now presents with delayed myelination and epilepsy since 6 months of age.

\section{Child has poor cry and is drowsy.}

Imaging: Multiple T2, FLAIR and Diffusion weighted hypointense and T1 isointense subependymal nodules along the lateral ventricles on both sides which suggest presence of calcified nodules.

Multiple cortical tubers with white matter migration lines were seen in bilateral supratentorial white matter.

There was moderate thinning of corpus callosum with mild frontal and temporal atrophy
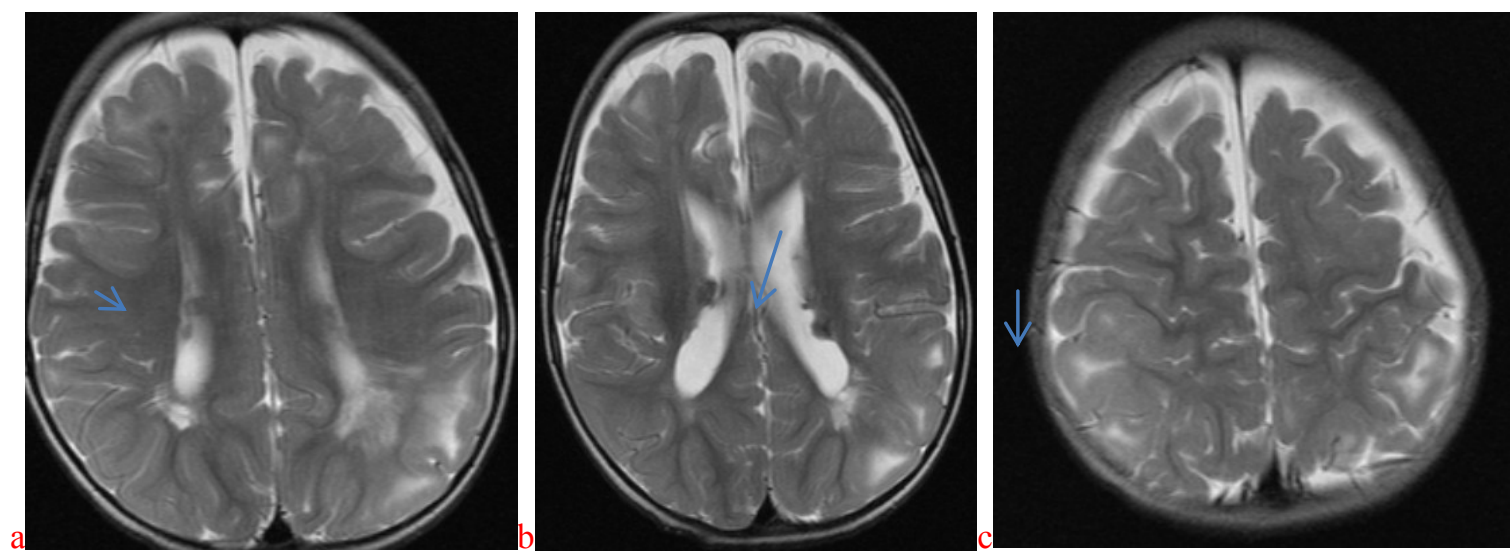

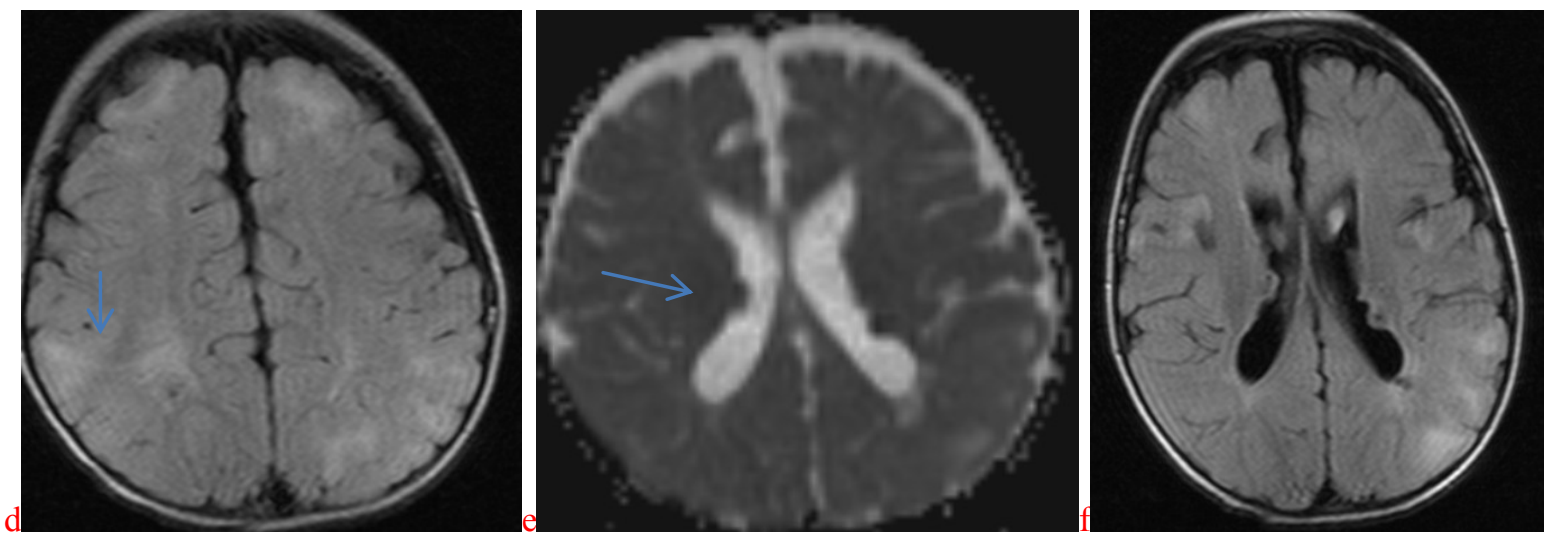

Fig 2 a,b ) T2 axial images show hypointense subependymal nodules ( arrows) c) T2 axial image shows hyperintense cortical tubers (arrows).d)FLAIR axial image shows white matter migration lines ( arrow).e) ADC image shows subependymal nodules ( arrow).f) FLAIR axial shows subependymal nodules, tubers and radiation lines .

\section{Case 3}

A 12 year old male born to non-consanguineous couple

Clinical profile:

Child presents with delayed milestones and severe mental retardation with epilepsy for the past 4 years.

Patient elder sibling had similar complaints

Imaging: MRI and CT revealed an extra-axial lobulated mixed intense mass adjacent to the right lateral ventricle.

There were multiple calcified subependymal nodules along the lateral ventricles on both sides.

Few cortical tubers with white matter migration lines were also seen in the supratentorial region.

Usg abdomen later revealed renal angiomyolipomas
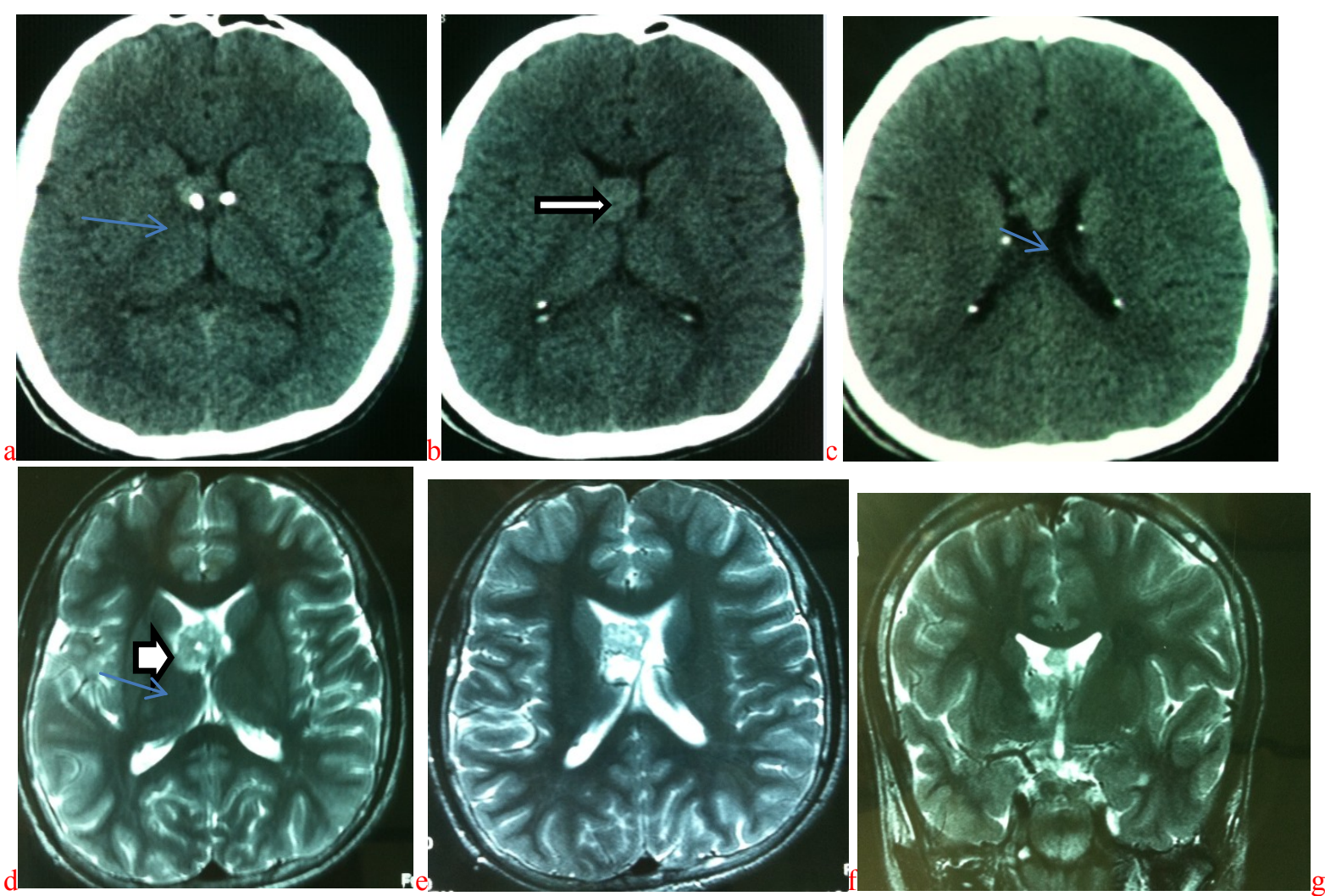

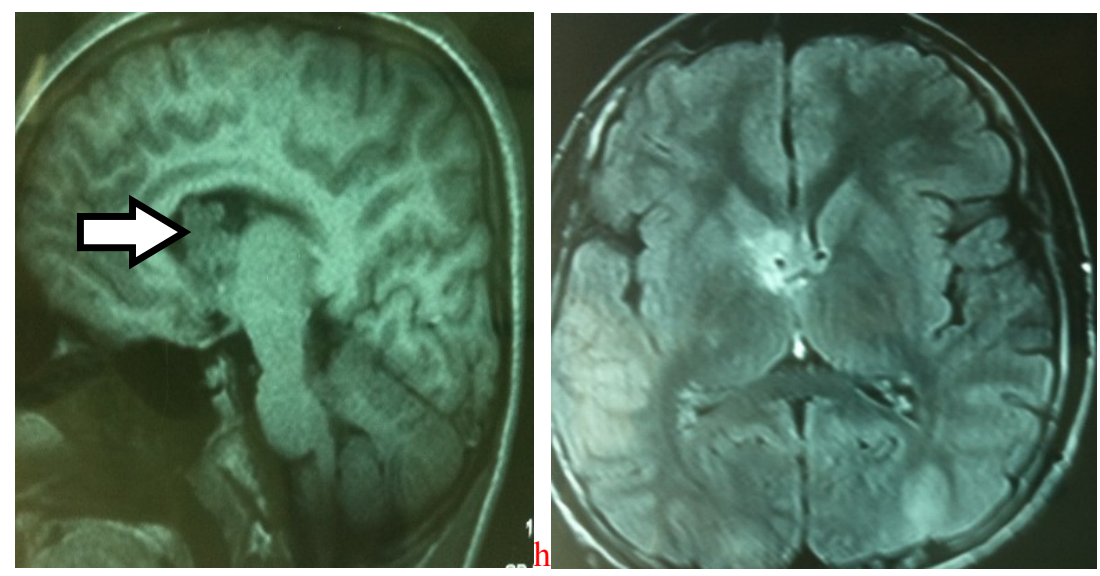

Fig 3 a,b,c) CT axial images show calcified subependymal nodules (arrows) and mixed density subependymal giant cell astrocytoma( block arrow)d,e,f,g,h) MRI T2 and FLAIR images shows subependymal giant cell astrocytoma(block arrow) and white matter migration lines(arrows)

\section{Discussion:}

Tuberous sclerosis complex (TSC) has also been called Bourneville or Bourneville-Pringle disease. The classic clinical triad of TSC consists of facial lesions ("adenomata sebaceum"), epilepsy, and mental retardation.

Tuberous sclerosis complex is a neurocutaneous syndrome characterized by the formation of non-malignant hamartomas and neoplastic lesions in the brain, heart, skin, kidney, lung, and other organs. It is associated with autism, seizures, and neurocognitive and behavioral disabilities. Because its clinical manifestations vary widely, establishing the diagnosis of tuberous sclerosis complex was particularly challenging prior to the advent of modern neuroimaging and genetic phenotyping.

Genetics: Approximately $50 \%$ of TSC cases are inherited and follow an autosomal dominant pattern. The other half represents de novo mutations and germline mosaicism.

Two separate genes are mutated or deleted in TSC: TSC1 and TSC2. TSC2 mutations are approximately five times as frequent as those affecting TSC1.

The TSC1 gene is located on chromosome 9q34 and encodes a protein called hamartin. The TSC2 gene is localized to chromosome 16p13.3 and encodes the tuberin protein. Mutations in either gene are identified in 75$85 \%$ of patients with TSC.

The TSC1/TSC2 protein dimer complex functions as a tumor suppressor. Hamartin/tuberin inhibits the complex signalling pathway called mammalian target of rapamycin (mTOR). Mammals possess only a single mTOR gene. The mTOR protein product is a component of two complexes, mTORC1 and mTORC2. Activation of either mTORC regulates protein synthesis and cell growth.

Mutations that lead to increased mTOR activation promote cellular disorganization, overgrowth, and abnormal differentiation that may result in tumorigenesis.

Presentation.TSC patients generally present within the first two decades of life. The most common skin lesions are hypomelanotic macules, which are ovoid depigmented areas with irregular margins that are best visualized by ultraviolet light (Woods lamp). These "ash leaf" spots are seen in over $90 \%$ of cases and may be the first visible manifestation of TSC. Other common cutaneous findings such as forehead plaques, shagreen patches, facial angiofibromas ("adenoma sebaceum"), and periungual fibromas usually do not appear until after puberty.

Diagnostic Criteria:

The clinical diagnosis of TSC is problematic because all cutaneous features are age dependent and may not become apparent until later in childhood. The classic triad of facial "adenomata sebaceum", seizures, and mental retardation is seen in only $30 \%$ of patients.

The various clinical features of TSC are designated as either major or minor features. Based on these features, the diagnosis is divided into definite, probable, and possible TSC. While DNA testing is useful for diagnosis and determining the causative mutation, approximately $30 \%$ of patients with definite TSC have negative results for TSC 1 and TSC2 mutations. 
MAJOR:

- Facial angiofibromas

- Non traumatic ungula or periungual fibromas

- Hypomelanotic macules

- Shagreen patches

- Multiple retinal nodular hamartomas

- Cerebral tubers

- Subependymal nodules

- Subependymal giant cell astrocytoma

- Cardiac rhabdomyoma,single or multiple

- Lymphangioleiomyomatosis

- Renal angiomyolipoma

MINOR:

- Multiple randomly distributed enamel pits in dental enamel

- Hamartomous rectal polyps

- Bone cysts

- Affected first degree relative

- Cerebral white matter migration lines

- Gingival fibromas

- Nonrenal hamartomas

- Retinal achromic patch

- Confetti skin lesions

- Multiple renal cysts

Definite: 2 major or 1 major plus 2 minor

Probable: 1 major plus 1 minor

Possible: 1 major or 2 or more minor features

\section{IntracranialManifestations:}

The four major pathologic features of TSC in the brain -cerebral tubers, subependymal nodules, white matter lesions, and subependymal giant cell astrocytoma

Three minor features which present with a lesser frequency than the major ones - parenchymal cysts, cerebellar lesions and vascular lesions.

Cerebral Tubers.Cerebral tubers or hamartomas are firm, whitish, pyramid-shaped, elevated areas of smooth gyral thickening, with or without central depressions that grossly resemble potatoes ("tubers").

Microscopically, cortical tubers consist of giant cells and dysmorphic neurons with foci of gliosis, disrupted lamination, and disordered myelin. Balloon cells similar to those seen in Taylor-type focal cortical dysplasia (FCD IIb) are also commonly found in tubers. Tubers do not undergo malignant transformation.

They are most common supratentoriarly, though around $15 \%$ may have in the cerebellum

The proportion that calcifies is not known but there is seen an increase in calcified tubers with increase in age , presenting around $50 \%$ in patients above 10 years of age

Neonatal and infantile cortical tubers are initially seen as hypodense cortical/subcortical masses within broadened and expanded gyri. The lucency decreases with age; tubers in older children and adults are mostly isodense with cortex this maybe attributed to the myelination of white matter. FLAIR images best depict parenchymal lesions in a myelinated brain.

Tubers are almost always located in the subcortical regions, separate from the overlying cortex. Cysts also are seen within the tubers in myelinated mature brains and represent a more aggressive phenotype. On MR perfusion most of the tubers appear hypoperfused.On MR spectroscopy the tubers showed mildly elevated choline and decreased NAA peaks with increased myo-inositol peaks on short TE (20-30 msec).

SubependymalNodules: Subependymal nodules (SENs) are located immediately beneath the ependymal lining of the lateral ventricles, along the course of the caudate nucleus. They are the most common intracranial manifestation.

SENs appear as elevated, rounded, hamartomatous lesions that grossly resemble candle guttering or drippings. They often calcify with increasing age. SENs along the caudothalamic groove adjacent to the foramen of Monro may undergo neoplastic transformation into subependymal giant cell astrocytoma. 
SENs are rarely calcified in the first year of life. Calcification in SENs increases with age. Eventually, 50\% demonstrate some degree of globular calcification. SENs typically do not enhance on CECT scans. An enhancing or enlarging SEN — especially if located near the foramen of Monro-is suspicious for SEGA.

They usually have increased diffusivity and reduced fractional anisotropy compared to the surrounding white matter.

White Matter Lesions. White matter (WM) lesions are almost universal in patients with TSC. They appear as foci of bizarre dysmorphic neurons and balloon cells in the subcortical WM and/or fine radial lines extending outward from the lateral ventricles.

These white matter foci contain areas of hypomyelination similar to those seen in cerebral tubers.

These lesions are usually missed on CT scans, on MR imaging they are usually seen to to course through the entire cerebral mantle.

Subependymal Giant Cell Astrocytoma (SEGA) - also known as subependymal giant cell tumor-is seen almost exclusively in the setting of TSC. Grossly, SEGAs appear as well-circumscribed solid intraventricular masses located near the foramen of Monro. SEGAs are WHO grade I tumors that often cause obstructive hydrocephalus but do not invade adjacent brain. While most SEGAs are unilateral, bilateral tumors occur in $10-15 \%$ of cases.

Typical microscopic features are large (not truly giant), plump cells that resemble astrocytes and/or ganglion cells in a fibrillar background. Tumor cell GFAP positivity varies, but most SEGAs are positive for neurofilament protein, neuron-specific enolase, and synaptophysin on immunohistochemistry.

Intratumoral calcifications are relatively common, but necrosis is rare. Mitoses are few, and the MIB-1 index is generally low.

Subependymal giant cell astrocytoma. While SEGAs can occur anywhere along the ventricular ependyma, the vast majority are found near the foramen of Monro. SEGAs are mixed signal intensity on both T1and T2WI. Virtually all enhance moderately strongly on T1 C+ scans.

SEGAs become symptomatic when they obstruct the foramen of Monro and cause hydrocephalus. Even large SEGAs rarely invade brain.

Parenchymal Cysts: An unknown number of patients with TSC show parenchymal cystic benign lesions, usually in the periventricular regions. There clinical significance is not yet certain.

CerebellarLesions: They are less common presenting around $15 \%$, when present they show similar features like in the cerebral hemispheres.

VascularLesions: These are very rare, and may present as aneurysms in the internal carotid and anterior cerebral artery territory, better appreciated on angiography.

\section{Conclusion}

Neuroimaging in a small series of patients with tuberous sclerosis and mental retardation and epilepsy revealed severely afflicted brains with more white matter anomalies, subependymal nodules, SGCA, transmantle dysplasias and cerebral tubers.

Neuroimaging plays a vital role in the diagnosis of the TSC,and is often a conclusive and characteristic , on most occasions not requiring further follow ups.

\section{References}

[1]. Katz JS et al: Intraventricular lesions in tuberous sclerosis complex: a possible association with the caudate nucleus. J NeurosurgPediatr. 9(4):406-13, 2012

[2]. Dobashi Y et al: Mammalian target of rapamycin: a central node of complex signaling cascades. Int J ClinExpPathol. 4(5):476-95, 2011

[3]. Hake S: Cutaneous manifestations of tuberous sclerosis. Ochsner J. 10(3):200-4, 2010

[4]. Takanashi J, Sugita K, Fujii K et-al. MR evaluation of tuberous sclerosis: increased sensitivity with fluid-attenuated inversion recovery and relation to severity of seizures and mental retardation. AJNR Am J Neuroradiol. 1995;16 (9): 1923 -8. AJNR Am J Neuroradiol (abstract) - Pubmed citation

[5]. Goh S, Butler W, Thiele EA. Subependymal giant cell tumors in tuberous sclerosis complex. Neurology. 2004;63 (8): 145761. Neurology (full text) - Pubmed citation

[6]. Umeoka S, Koyama T, Miki Y et-al. Pictorial review of tuberous sclerosis in various organs. Radiographics. 28 (7): e32. doi:10.1148/rg.e32 - Pubmed citation

[7]. Logue LG, Acker RE, Sienko AE. Best cases from the AFIP: angiomyolipomas in tuberous sclerosis. Radiographics. 23 (1): 241-6. doi:10.1148/rg.231025109 -Pubmed citation

[8]. Roach ES, Gomez MR, Northrup H. Tuberous sclerosis complex consensus conference: revised clinical diagnostic criteria. J. Child Neurol. 1998;13 (12): 624-8.doi:10.1177/088307389801301206 - Pubmed citation

[9]. Umeoka S, Koyama T, Miki Y et-al. Pictorial review of tuberous sclerosis in various organs. Radiographics. 28 (7): e32. doi:10.1148/rg.e32 - Pubmed citation

[10]. Bell DG, King BF, Hattery RR et-al. Imaging characteristics of tuberous sclerosis. AJR Am J Roentgenol. 1991;156 (5): 1081 6. AJR Am J Roentgenol (citation) - Pubmed citation 
[11]. Kalantari BN, Salamon N. Neuroimaging of tuberous sclerosis: spectrum of pathologic findings and frontiers in imaging. AJR Am J Roentgenol. 2008;190 (5): W304-9. doi:10.2214/AJR.07.2928 - Pubmed citation

[12]. Kwiatkowski DJ, Whittemore VH, Thiele EA. Tuberous Sclerosis Complex, Genes, Clinical Features and Therapeutics. WileyVCH. (2010) ISBN:3527322019. Read it at Google Books - Find it at Amazon

[13]. Barkovich AJ. Pediatric neuroimaging. Lippincott Williams \& Wilkins. (2005) ISBN:0781757665. Read it at Google Books Find it at Amazon

[14]. Atlas SW. Magnetic resonance imaging of the brain and spine. Lippincott Williams \& Wilkins. (2008) ISBN:078176985X. Read it at Google Books - Find it at Amazon

[15]. Bernauer TA. The radial bands sign. Radiology. 1999;212 (3): 761-2.doi:10.1148/radiology.212.3.r99se17761 - Pubmed citation 